\title{
Study on the Correlation between the Expression of Serum Ferritin and Gestational Diabetes Mellitus
}

\author{
Weiwei Li1, Zhijuan Sun ${ }^{2 *}$ \\ ${ }^{1}$ Department of Clinical Laboratory, Qingdao Sanatorium of Shandong Province, Qingdao, China \\ ${ }^{2}$ Department of Clinical Laboratory, Qingdao No. 9 People’s Hospital, Qingdao, China \\ Email: *scientific1023@163.com
}

How to cite this paper: Li, W.W. and Sun, Z.J. (2020) Study on the Correlation between the Expression of Serum Ferritin and Gestational Diabetes Mellitus. Open Journal of Obstetrics and Gynecology, 10, 1135-1140.

https://doi.org/10.4236/ojog.2020.1080106

Received: July 5, 2020

Accepted: August 24, 2020

Published: August 27, 2020

Copyright ( 2020 by author(s) and Scientific Research Publishing Inc. This work is licensed under the Creative Commons Attribution International License (CC BY 4.0).

http://creativecommons.org/licenses/by/4.0/

(c) (i) Open Access

\begin{abstract}
Objective: To explore the relationship between serum ferritin (SF) and gestational diabetes mellitus (GDM), for providing new ideas to the prevention and treatment of GDM. Methods. All the pregnant women were selected in Yinan Maternal and Child Health-Care Hospital from December, 2015 to March, 2018 when they were having routine prenatal examination, According to the diagnostic criteria of GDM, 72 patients with GDM were selected as the case group and 72 normal pregnant women were randomly selected as the control group. Fasting venous blood was drawn from all subjects during the first trimester of pregnancy (11 - 13 weeks) and the second trimester of pregnancy (24 - 28 weeks). Fasting plasma glucose was measured by glucose oxidase assay and the expression level of SF was determined by electrochemical method. The application value of SF in GDM diagnosis was evaluated by ROC curve. Results. The levels of SF in the case group at early and middle stages are $49.6 \pm 18.8$ $(\mu \mathrm{g} / \mathrm{ml})$ and $39.8 \pm 21.5(\mu \mathrm{g} / \mathrm{ml})$, which re $39.4 \pm 15.2(\mu \mathrm{g} / \mathrm{ml})$ and $32.2 \pm 17.6$ $(\mu \mathrm{g} / \mathrm{ml})$ in the control group. The levels of SF in the case group were higher than those in the control group $(p<0.05)$ at early and middle stages. The curve (AUC) of SF level in the diagnosis of GDM was 0.895, $p<0.001$; the Youden index was 0.651 and the optimum threshold was $38.6 \mathrm{ng} / \mathrm{ml}$, with a sensitivity of $97.8 \%$ and a specificity of $67.3 \%$. Conclusion: The expression level of SF in early pregnancy is correlated with the occurrence of GDM, which may be an important indicator for the prevention and monitoring of GDM.
\end{abstract}

\section{Keywords}

Serum Ferritin, Gestational Diabetes Mellitus, Correlation 


\section{Introduction}

The gestational diabetes mellitus (GDM) refers to different degrees of hyperglycemia resulting from abnormal glucose tolerance and diabetes during the gestation period, but the glycometabolism is normal before gestation. According to the report, the morbidity of GDM in China has reached $18.9 \%$ in 2014, and tends to have a significant growth due to the influences of many factors such as age of the pregnant woman [1]. We shall pay high attention to pregnant women with GDM because the glycometabolism may result in many adverse outcomes such as infection, premature birth, and dystocia due to its complex process and high risk [2] [3]. Many researchers have indicated that increase of iron element level in serum can result in changes in the blood glucose and have a correlation with insulin resistance [4] [5]. Serum ferritin (SF) is one of the proteins with the highest iron content in the body. It can directly reflect the iron reserves in the body and is a sensitive indicator for evaluating iron deficiency or overload. In recent years, studies have found that abnormal iron metabolism is closely related to the occurrence and development of T2DM. Related, high SF level is an independent risk factor for T2DM. The serum ferritin (SF) is an important index that reflects the content of iron element in body, while high levels of serum iron significantly increase the risk of GDM [6]. At present, there are few researches about correlation between SF and GDM; therefore, we have measured SF expression level at early and middle pregnancy and discussed its correlation with GDM in this research, laying theoretical foundation for early prevention and clinical treatment for GDM.

\section{Data and Methods}

\subsection{General Data}

We have selected 72 pregnant women with GDM who have made routine examination in obstetrical department of Hospital from December 2015 to March 2018 as a case group, and randomly selected 72 normal pregnant women as a control group. These pregnant women with GDM must be diagnosed strictly in accordance with Standards for GDM Diagnosis issued by the American Diabetes Association (ADA) in 2013 [7] and selected as GDM group during the second trimester $\left(24^{\text {th }}\right.$ to $28^{\text {th }}$ week). Besides, all objects must sign the Informed Consent Form. The exclusion standards include definite diagnosis of GDM during progestational period, anemia during gestation, ferro-therapy, and trauma, etc.

\subsection{SF Measurement}

We have drawn venous blood of all pregnant women with an empty stomach during the first trimester ( $11^{\text {th }}$ to $13^{\text {th }}$ week $)$ and the second trimester $\left(24^{\text {th }}\right.$ to $28^{\text {th }}$ week) to measure SF and blood glucose concentration respectively with DXI800 automatic immune diagnosis device (Beckman, USA). All operations have been made strictly in accordance with general SOPs. 


\subsection{Statistical Treatment}

We have used the software SPSS20.0 for statistical analysis; denoted measurements by "mean + standard deviation" $(\bar{x} \pm s)$ and enumeration data by utilization ratio; conducted t-test for mean comparison and $\chi^{2}$ test for comparison between above two groups; and evaluated value of SF in diagnosing early GDM by ROC (receiver operating characteristic curves). $P<0.05$ indicates statistical difference.

\section{Results}

\subsection{General Data Comparison}

See Table 1. The comparison between the case group and the control group in age, number of pregnancies, gestational weeks, and BMI of pregnant women indicates no statistical difference $(P>0.05)$.

\subsection{Comparison between the Case Group and the Control Group in SF ( $\mu \mathrm{g} / \mathrm{ml})$ Level in Different Periods of Pregnancy}

See Table 2. According to the comparison between the case group and the control group in SF expression level of peripheral blood (PB) in different periods of pregnancy, SF of the case group is higher than the control group, indicating statistical difference $(p<0.05)$.

\subsection{Analysis for ROC between SF and GDM in Different Periods of Pregnancy}

We have used ROC for analyzing values of clinical diagnosis for GDM, as shown in Figure 1. The AUC in diagram of ROC between SF and GDM at the first

Table 1. Comparison between the case group and the control group in general data $(\bar{x} \pm s)$.

\begin{tabular}{cccccc}
\hline Groups & $\mathrm{N}$ & Age (years) & Week & Times & BMI $\left(\mathrm{Kg} / \mathrm{m}^{2}\right)$ \\
\hline Case Group & 72 & $29.86 \pm 3.68$ & $12.56 \pm 1.18$ & $1.95+0.50$ & $23.15 \pm 7.28$ \\
Control Group & 72 & $29.01 \pm 4.12$ & $12.21 \pm 1.25$ & $2.0+0.75$ & $21.18 \pm 5.21$ \\
$\mathrm{t}$ & & 1.382 & 0.862 & 0.49 & 0.156 \\
$p$ & & $0.151^{\star}$ & $0.560^{\star}$ & $0.624^{\star}$ & $0.916^{\star}$ \\
\hline
\end{tabular}

Notes: ${ }^{*}: p>0.05$ indicates no statistical difference compared to the control group.

Table 2. Comparison between the case group and the control group in SF ( $\mu \mathrm{g} / \mathrm{ml})$ level in different periods of pregnancy.

\begin{tabular}{cccc}
\hline Groups & $\mathrm{N}$ & $11-13$ Week & $24-28$ Week \\
\hline Case Group & 72 & $49.6 \pm 18.8$ & $39.8 \pm 21.5$ \\
Control Group & 72 & $39.4 \pm 15.2$ & $32.2 \pm 17.6$ \\
$\mathrm{t}$ & & 8.260 & 2.366 \\
$p$ & & $0.010^{*}$ & $0.025^{\star}$ \\
\hline
\end{tabular}

Notes: ${ }^{*}: p<0.05$ indicates statistical difference compared to the control group. 


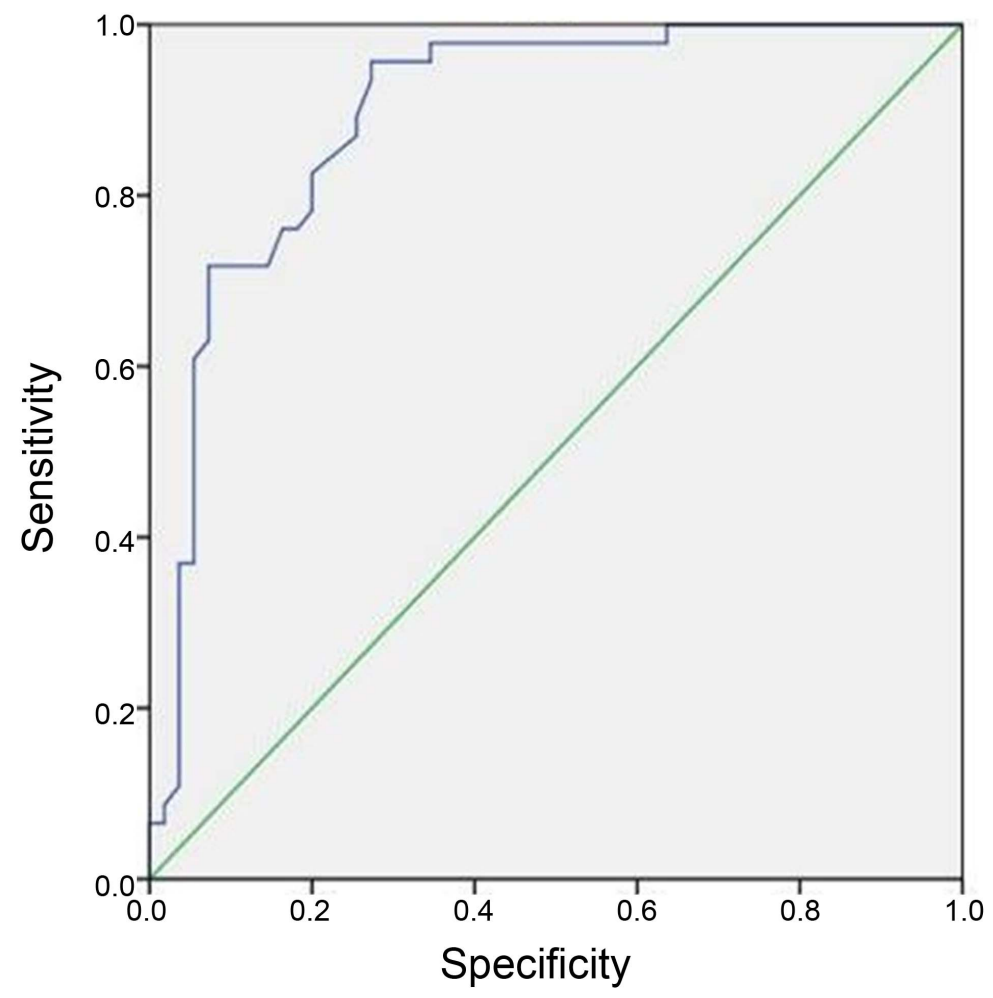

Figure 1. Analysis for ROC between SF and GDM in different periods of pregnancy.

trimester is $0.895(p<0.001)$. In ROC, Youden index is 0.651 and SF critical value is $38.6 \mathrm{ng} / \mathrm{ml}$; meanwhile, values of sensitivity and specificity are $97.8 \%$ and $67.3 \%$ respectively.

\section{Discussion}

GDM is one of frequent complications in obstetrics department, of which pathogenesis has not been explained completely. Many researches indicate that pathogeny of GDM is influenced by numerous factors, including familial inheritance, insulin resistance, metabolic and autoimmune disorders, and some risk factors such as age, weight, and obesity of pregnant women [8] [9] [10]. There is no significant difference between GDM case group and control group in age, the number of pregnancies, gestational weeks, and BMI of pregnant women, but significant difference in SF expression level, indicating possibly high correlation between SF and pathogeny of GDM.

With complex structure, SF is large-scale colloid ferrous globulin which is not only a major ferrous storage globulin in body, but also an important means of transport for Fe. At present, pathogenesis has not been explained completely, but many researchers have indicated that SF plays an important role in the pathogenic process of GDM. According to the research, as Fe level in the body of a pregnant women increases, morbidity of GDM also increases, which may be based on the inflammatory reaction. Besides, there is a significant correlation between SF and inflammatory factor in expression level [11] [12]. Expressions 
between SF and inflammatory factor are mutually stimulated, where inflammatory factor induces synthesis of SF, while SF with high expression acts on islet cells to generate insulin resistance, resulting in rise of blood glucose concentration. However, blood glucose with high concentration also results in a large number of inflammatory substances by cells in the body, which aggravates GDM's damage on the body [13].

ROS is a series of reactive oxygen species generated by aerobic cells in the metabolic process, which can induce cell apoptosis or tissue damage through oxidative stress reaction of cells. The mitochondria and lysosome of organelle in the islet cells have rich ROS of which generation and elimination under physiological conditions are dynamically balanced. When SF storage in the body has reached a high level, redundant ferric ions can catalyze excessive ROS in the body, resulting in insulin resistance, damage on islet cells and restriction for insulin synthesis and secretion through oxidative stress reaction. As a result, this has further accelerated the occurrence and evolution of GDM [14] [15]. The results from ROC indicate that AUC in diagram of ROC between SF and GDM in the first trimester is $0.895(p<0.001)$. In ROC, Youden index is 0.651 and SF critical value is $38.6 \mathrm{ng} / \mathrm{ml}$; meanwhile, values of sensitivity and specificity are $97.8 \%$ and $67.3 \%$ respectively, indicating important values of SF measurement for early GDM screening.

There are some shortcomings to this study. We only detected the index of serum ferritin, and the specificity is not high. If we can add some indicators and make the ROS curve together, it will greatly improve the detection rate of early gestational diabetes.

\section{Conclusion}

There is a certain correlation between GDM and expression level of SF. Therefore, detecting the expression levels of SF at early pregnancy and middle pregnancy will be of great value for the prevention and treatment of gestational diabetes.

\section{Conflicts of Interest}

The authors declare no conflicts of interest regarding the publication of this paper.

\section{References}

[1] Erjavec, K., Poljicanin, T., Matijevic, R., et al. (2016) Impact of the Implementation of New WHO Diagnostic Criteria for Gestational Diabetes Mellitus on Prevalence and Perinatal Outcomes: A Population-Based Study. Journal of Pregnancy, 2016, Article ID: 2670912. https://doi.org/10.1155/2016/2670912

[2] Wei, Y.M., Liu, X.Y., Shou, C., et al. (2019) Value of Fasting Plasma Glucose to Screen Gestational Diabetes Mellitus before the 24th Gestational Week in Women with Different Pre-Pregnancy Body Mass Index. Chinese Medical Journal (EngI), 132, 883-888. https://doi.org/10.1097/CM9.0000000000000158 
[3] Leng, J., Shao, P., Zhang, C., et al. (2015) Prevalence of Gestational Diabetes Mellitus and Its Risk Factors in Chinese Pregnant Women: A Prospective Population-Based Study in Tianjin, China. PLoS ONE, 10, e0121029. https://doi.org/10.1371/journal.pone.0121029

[4] Alam, F., Shahbaz, H., Khuwaja, S., et al. (2017) Implication of Soluble Transferrin Receptor and Ferritin Ratio in Gestational Diabetes. International Journal of Diabetes in Developing Countries, 38, 42-46. https://doi.org/10.1007/s13410-017-0571-4

[5] Achuthan, A. and Mageswari, U. (2017) Correlation between Serum Free Iron, Glycated Hemoglobin and Insulin Resistance in Uncontrolled Type-2 Diabetic Patients. National Journal of Physiology, Pharmacy and Pharmacology, 7, 642. https://doi.org/10.5455/njppp.2017.7.1234002032017

[6] Chen, W., Li, L., Rina, S., et al. (2018) Hemoglobin Levels during the First Trimester of Pregnancy Are Associated with the Risk of Gestational Diabetes Mellitus, Pre-Eclampsia and Preterm Birth in Chinese Women: A Retrospective Study. BMC Pregnancy \& Childbirth, 18, Article No. 263. https://doi.org/10.1186/s12884-018-1800-7

[7] American Diabetes Association (2013) Standards of Medical Care in Diabetes-2013. Diabetes Care, 36, S11-S66. https://doi.org/10.2337/dc13-S011

[8] Muller, P.S. (2018) Analysis of Effects of Most Influential Risk Factors on Gestational Diabetes Mellitus. Investigación Clínica, 59, 376-385.

[9] Xiang, A.H., Kawakubo, M. and Trigo, E. (2010) Declining $\beta$-Cell Compensation for Insulin Resistance in Hispanic Women with Recent Gestational Diabetes Mellitus. Diabetes Care, 33, 396-401. https://doi.org/10.2337/dc09-1493

[10] Wang, Y. and Luo, B. (2019) Risk Factors Analysis of Gestational Diabetes Mellitus Based on International Association of Diabetes Pregnancy Study Groups Criteria. Journal of Southern Medical University, 39, 572-578.

[11] Wang, L., Li, H.-L., Liu, A.-N., et al. (2015) Changes and Significance of Serum Inflammatory Factors in Type 2 Diabetes Patients. Clinical Medicine, 3, 23-24.

[12] Guo, W., Wang, H., Liu, Q., et al. (2018) Analysis of the Correlation of Gestational Diabetes Mellitus and Peripheral Ferritin with Iron Levels in Early Pregnancy. Minerva Endocrinologica, 44, 91-96. https://doi.org/10.23736/S0391-1977.18.02734-7

[13] Fu, S.M., et al. (2016) The Relationship between Body Iron Status, Iron Intake and Gestational Diabetes: A Systematic Review and Meta-Analysis. Medicine, 95, e2383.

[14] Klisic, A., Isakovic, A., Kocic, G., et al. (2018) Relationship between Oxidative Stress, Inflammation and Dyslipidemia with Fatty Liver Index in Patients with Type 2 Diabetes Mellitus. Experimental and Clinical Endocrinology \& Diabetes, 126, 371-378. https://doi.org/10.1055/s-0043-118667

[15] Eren, E., et al. (2016) Relationship between Thioredoxin and Thioredoxin-Binding Protein in Patients with Gestational Diabetes Mellitus. Journal of Maternal Fetal \& Neonatal Medicine, 30, 164-168. https://doi.org/10.3109/14767058.2016.1163685 